\title{
THE CONJUNCTION OF NON-LIVING AND LIVING IN HUMAN SYSTEMS: WHY DO NOVELTIES EMERGE?
}

\author{
R.M. PULSELLI, F.M. PULSELLI \& N. MARCHETTINI \\ Department of Chemical and Biosystems Sciences, University of Siena, Siena, Italy.
}

\section{ABSTRACT}

The large picture and the ideal of total control upheld by traditional economics are unlikely to obtain complete answers in the increasing complexity of the socioeconomic system with its various manifestations, such as globalisation and environmental depletion. The reason why the economy does what it does and why we pay attention to it gets lost along the way. In other words, the instruments of economics are inadequate for the complex socioeconomic context in which we live. To illustrate this concept as a starting point for new foundations for economic disciplines, we present an unusual scientific discussion inspired by a fantasy thriller film of 1997 titled Cube.

Keywords: adaptation, co-evolutionary processes, complexity, economic system, Gödel's theorem, irreducibility, perturbation, self-organization, society.

\section{INTRODUCTION}

The economic system consists of a set of interacting parts. Its aleatory consistency is supported by regulations, laws, decrees, constrictions and codes. Besides this rational system, economics presupposes exchange, action and interaction that can be expressed in terms of money and assumes the existence of a dynamic factor (physical reference system, ecosystem, subjects that exchange) in which humans are the dominant component. An economic system is therefore expressed as the interaction of a contrived rational component (laws, regulations, principles) with a vital dynamic human component (free will, personality, emotions, supply and demand, habits and customs). In the economic system, a set of abstract, mechanical, lifeless structural elements combines with a social component, a community of persons, individuals, groups, companies and other dynamic, vital types of organisms. This conjunction of non-living and living offers an interpretative key for the economic system, which theoretically acquires all the dynamic characteristics of a living organism.

$$
\begin{aligned}
& \text { Non-living things } \\
& \text { (rules, codes })
\end{aligned} \quad+\quad \begin{gathered}
\text { Living agents } \\
(\text { society, individuals })
\end{gathered} \quad=\quad \begin{gathered}
\text { Living system } \\
(\text { economic system })
\end{gathered}
$$

A system containing the vitality of its living elements absorbs their properties (rational and emotional) and makes them into a whole. It is difficult to determine because it adapts its behaviour by diffuse and radical structural changes, even in response to very small changes. It is also difficult to control by mechanistic laws.

\section{THE PLOT: CHARACTERS' BEHAVIOUR AMONG CONSTRAINING RULES}

The plot of Cube [1] is simple. Six people are confined to a box full of traps, like a Rubik's cube. All known space is represented by the cube; the time they have to get out of the trap is sanctioned by physiological needs. Three days and three nights without eating and drinking are enough to threaten anyone's survival. A perfect biological deadline.

The plot of the film can be considered from many viewpoints and lends itself to many interpretations. Six people find themselves in a new and hostile context. It is interesting to observe how they 
adapt to their aseptic microcosm. Resorting to mathematical logic, the way out is discovered by deciphering the rules of the game and solving the riddle. The Cube is a system that obeys reducible linear laws which are therefore determinate and predictable. At the same time, however, there are six personalities that soon manifest bizarre, unpredictable behaviours under the pressure of a hostile environment. The process of natural selection in a closed box creates unexpected situations. The character who seems the strongest is overcome by emotion and cannot control the situation and adapt - he loses his head. An autistic boy has the qualities necessary to survive aggression by the Cube and to lead the others out, and manages well with their help. From a purely scientific viewpoint, the plot of the film revolves around adaptation and selection. The strength of the film is the combination of few essential elements: an aseptic environment and a group of strangers. A box (non-living) that obeys mechanical laws combined with the personalities and emotions of the characters (living) gives rise to an intense sequence of unexpected events and emerging novelties. The Cube can therefore be a metaphor for the system of rules, mechanisms and cogs of the economy, which is assembled on the basis of rational criteria and acts like a machine. The rooms in the Cube contain different obstacles, as different rules and methods are applied in different states and regions of the world. The characters cannot escape from the Cube just as society and local communities cannot escape from the global economic system. Society can only exist inside the Cube. There are no alternatives for anyone, not even for the tiny community of Mapuche in southern Patagonia. The entire story takes place in the Cube and the behaviour of individuals creates understanding or disagreement, separation or conflict. The assembled box follows its inexorable course and the prisoners succumb to the rigidity of its structure. Paradoxically, the autistic boy, who can process information in a linear manner, as required by the Cube's mechanics, and is helped by the others, is the one who finds the way out. The narrow margins of salvation allowed by the Cube, which represents the rigid schemes of the economy, as we see it, lets few emerge, and the characters' emotions, diversity and thoughts are evidently irreconcilable with cubism. The Cube is a law unto itself and unable to respond to the living component of the society it so rigidly controls. In such a situation, the six prisoners have to learn its rules and take advantage of what they know in order to escape. Faced with an inexorable machine, the instinct to survive leads them into falls, crises, competitivity, iniquity, disagreement and loss. The result is a complex story, a devastating plot.

\section{EMERGENCE OF NOVELTIES}

The external observer asks who built the Cube and why? An explanation springs from sharp reasoning and fine logic (in fact, this is just a personal speculation by the authors of this article on the basis of little information in the plot of the film). When one of the characters comes to the dramatic realisation that he was one of the designers of a section of the cube, an important clue is revealed. What is the Cube? The Cube is the result of many unrelated processes, small pieces commissioned by an unknown society and conceived by specialists who do not know each other, the overall design or the final result of their work.

Who built the Cube? A possible answer is everybody and nobody. In other words, the process of formation of the Cube could be a complex process that was activated, ran its course and concluded autonomously by a fatal combination of choices and chance. The characters would therefore be the unfortunate victims of an independent system larger than themselves.

The process of construction of the Cube that we are discussing simulates the evolution of a complex system having uncontrolled outcomes and may not be science fiction. The construction of a Cube is not an unlikely or improbable event in real life. The honest work of the engineer imprisoned in the Cube (irony of fate) with the other characters due to his high specialisation, does not necessarily mean that he knows the final aim of the product, whether it be a children's hospital or a war machine 
(or a cubic prison). His work is merely to exercise his specific skills to perfection. Direct communication between the parts of the process is not required. Certain parts of the process may have interacted and become a spontaneous process or system by a series of expedients and favourable circumstances. This is possible, especially when the immediate task of the parts is to find out how to receive an input from the system, make a transformation and get the system to make an output, at the same time making a profit. This description is familiar in modern economics. The mechanism, uncontrolled by any observer with an overall view of the system, is a possible description of the merely utilitarian cause that generates monsters such as the Cube. It is not the sleep of reason but the dream of reason that creates monsters [2].

To demonstrate this hypothesis, we have to use a specific science which sustains that the systemprocess exists and that it can take a recognizable form different from the identity of any of its parts. This concept was clearly expressed by Pascal, 'The whole is more than the sum of its parts', taken up by Von Bertalanffy [3] and others, and forms a basis of modern chaos and complex systems theory. In a complex system, when parts become constituents of a whole, they acquire meaning and an aim that they previously did not have. In such cases, the organisation of a system emerges spontaneously as added information that is not available from observation of the single parts.

Atoms tend towards a state of minimum energy by forming chemical bonds and becoming ordered in molecules. Organisms adapt to each other by a process of co-evolution and constitute an ecosystem. These are examples of how groups of agents that seek their own consistency and adapt to each other may transcend themselves and acquire collective properties, such as life and thought, which do not exist at the individual level [4].

This phenomenon is generally explained as the concept of self-organisation. Organisation emerges spontaneously, as if it were the system itself to choose it from many possibilities [5]. Each element of a whole follows its immediate personal interest, adapting its behaviour to that of nearby elements through small adjustments. This phenomenon is indicated as the law of minimum resistance. In complex systems, these small processes of adaptation are constantly present and compensate each other through repeated feedbacks, maintaining the system in a steady state in time. The presence of interactions between the parts of the system may, however, also cause sudden changes in the system in response to small perturbations induced by a new situation or by ambient changes [6]. Small reactions to new stimuli may affect much or all of the system, causing non-linear amplifications (positive feedback) and reorganisation of the whole. Nicolis [7] summed up these phenomena: 'Organisation emerges on a macroscopic spatiotemporal scale which is many times greater than the microscopic interactions between the elements'.

Such systems enjoy properties that cannot be predicted from reductive observation of their components. The whole system must be observed in order to apprehend its behaviour, because every part of the system depends on the others by direct and indirect connections. The presence of irreducible systems is coherent with Gödel's theorem, which states that it is impossible to give a detailed, complete and comprehensible description of the world. Most natural systems are irreducible, which places strong restrictions on the intrinsic reductionism of science. According to Gödel's theorem, the properties of order and emergence cannot be observed or known from inside the system, only by an external observer [8].

The more complex and characterised by interdependence of its parts (often highly specialised) a system is, the more likely it is that effects, which may be due to local phenomena, are felt on a large scale. Collective behaviour with new properties, which elude the control and understanding of individuals, may emerge as an effect of variations in relations between the different (social, economical, technological, physical) components of a system. For example, a variation in the physical environment, adoption of a system of rules or a slackening market may redefine the behaviour of a community. 
Considering the above, we can assume that the Cube is a set of mechanisms produced by the economic system and contemporary society. In the name of growth and progress, the economy and technology advance inexorably, sustained by research and false well-being, on a path that does not envisage any limits. The pursuit of immediate profit promoted by the Western model is increasingly divorced from an overall view of the common good, and thus from the real interests of individuals, from real well-being. This presumed common goal has become ambiguous and not in everyone's interests, but only in the interests of some.

According to the view of Adam Smith, by pursuing their personal interest, individuals automatically and unknowingly act in the interest of all, as if guided by an invisible hand. The theory of complexity shows that this concept is completely untrue. It is difficult to imagine the effects of the work of a Wall Street broker, who operates directly on the cogs of the economic system, when he celebrates a day of high profits in a bar in Greenwich Village. In extreme cases, his action may mean the prosperous survival of a company or the closure of a factory, the ruin of a community and the destitution of factory employees.

The metaphor of the Cube suggests that the consolidated economic system is out of control. Many solid facts suggest the same, by the very mechanism that created the Cube. The disproportionate distribution of planetary wealth, the increasing gulf between rich and poor, the great financial crashes, the economic crises, public debt, global environmental crisis, the effects of global warming and climate change, depletion of water resources and devastating wars are only some of the mechanisms represented by the metaphor of the Cube. They are results of a system that obeys its own laws and eludes all control. We wish that society never built the Cube and we wish there were no wars, but it is not easy to understand which complex mechanisms of the economic system underlie a war, which combinations of personal interest, self-organising phenomena and non-linear amplification involve thousands of persons, despite themselves, in futureless conflicts. If we could control these complex mechanisms, we could avoid harming ourselves, we could arrest the building of small cubes at any moment and we could dismantle the walls of the global Cube in which we live.

\section{CONCLUSION}

The mechanics of traditional economics configures a system that risks being a law unto itself, heedless of community and individual well-being and inclined to let them succumb. The contradictions of the economic system are the basis of the Cube, which represents the set of mechanisms that regulate society's behaviour and are not always knowingly determined. The Cube is the unexpected product of a process generated autonomously in a complex system, as are the social and economic systems. In these systems, in turn, interactions between dynamic elements and static normative apparatus generate phenomena that individuals cannot control and that are not easily arrested, such as war or the current environmental crisis. The greenhouse effect and fear of terrorism afflicting the entire human race are results of a system of rules and behaviours adopted by the global village which in turn impose new constrictions on the village (think of the urgent need to reduce emissions of greenhouse gases in defence against climate change and blanket security measures in defence against the risk of terrorist attacks). The Cube is a set of rules and mechanisms determined by contemporary society, but their interactions with living agents are not easy to determine and cause feedbacks which affect society in many complex plots.

Faced with such striking contradictions, traditional economics is a profoundly inadequate system of thought plagued with evident difficulties. The more power it acquires, the less able it is to govern this power. It denies responsibility for the social, environmental and cultural changes caused by its processes of growth. The economic system rests on unstable foundations as it pursues its one-way course towards infinity, but it always comes back to itself, in the name of deceptive growth, like the 
famous stairs of Escher, an illusory figure conceived to trick the mind. Faced with the need to investigate the world and the enormous variety of organisational, regulatory and adaptive solutions encountered in social dynamics, nature and life, economics is forced to change the type of questions it was accustomed to posing. Humankind has to urgently tackle great problems that threaten the survival of the species, that transcend the intrinsic utility of the instruments of traditional economics and reductionist science.

The sciences of evolutionary processes clamour to investigate concatenated and non-linear processes that cause novelties to emerge in systems. Economics has to find new, more flexible instruments, more suitable for describing the evolution of the system and its properties.

\section{REFERENCES}

[1] Cube, Director Vincenzo Natali, Trimark Pictures, Canada, 1997.

[2] Tiezzi, E., Beauty and Science, WIT Press: Southampton, 2005.

[3] Von Bertalanffy, L., General System Theory, Penguin Books: Harmondsworth, 1972.

[4] Waldrop, M.M., Complexity: The Emerging Science at the Edge of Order and Chaos, Simon and Schuster: New York, 1992.

[5] Bertuglia, C.S. \& Staricco, L., Complessità Autorganizzazione Città, Franco Angeli: Milano, 2000.

[6] Pulselli, R.M., Ratti, C. \& Tiezzi, E., City out of chaos: social patterns and organization in urban systems. International Journal of Ecodynamics, 1(2), pp. 126-135, 2006.

[7] Nicolis, G., Physics of far-from-equilibrium systems and self-organization. The New Physics, ed. P. Davies, Cambridge University Press: New York, 1989.

[8] Tiezzi, E., Steps Towards an Evolutionary Physics, WIT Press: Southampton, 2006. 